\title{
Requerimento térmico e características físico-químicas de diferentes variedades de videira em São Joaquim, SC
}

\author{
João Felippeto' ${ }^{1}$ Vinícius Caliari², Emílio Brighenti ${ }^{3}$ e Cristiano Nunes Nesi ${ }^{4}$
}

\begin{abstract}
Resumo - O objetivo deste trabalho foi caracterizar a fenologia, o requerimento térmico e os indicadores de maturação das bagas, de oito variedades viníferas durante uma série histórica entre 2013/14 a 2019/20 nas condições de São Joaquim, SC. As condições térmicas foram expressas em graus-dia (GD) correlacionando esses valores com a evolução dos subperíodos fenológicos. As bagas foram submetidas às análises de acidez total, pH e SST. Em média, o ciclo vegetativo das variedades foi de 200 dias, sendo necessários, durante esse período, 1.369 graus-dia. As variedades produziram uvas adequadas para a elaboração de vinhos finos, com destaque qualitativo para a 'Rebo' dentre as tintas e 'Manzoni Bianco' dentre as brancas.
\end{abstract}

Termos para indexação: fenologia; graus-dia; vinhos de altitude; videiras.

\section{Thermal requirement and physicochemical characteristics of different grape varieties in São Joaquim-SC}

Abstract - The objective of this work was to characterize the phenology, the thermal requirement and the maturation indicators of the berries, of eight wine varieties during a historical series between 2013/14 to 2019/20 in the conditions of São JoaquimSC. The thermal conditions were expressed in degrees-day (GD) correlating these values with the evolution of the phenological subperiods. The berries were subjected to analyzes of total acidity, $\mathrm{pH}$ and SST. The average vegetative cycle of the varieties was 200 days, being necessary, during this period, 1369, degree-days. The varieties produced grapes suitable for the production of fine wines, with a qualitative highlight for 'Rebo' among reds and 'Manzoni Bianco' among white ones.

Index terms: phenology; degree days; altitude wines; vines.

\section{Introdução}

A vitivinicultura brasileira tem ampliado a sua área de produção para além das regiões mais tradicionais, como a Serra Gaúcha e a Campanha Meridional do Rio Grande do Sul (RADÜZ et al., 2015). No contexto brasileiro, outras regiões têm merecido notoriedade em função da excelência dos seus produtos vitivinícolas. Esse é ocaso da região de São Joaquim, que possui uma atividade vitivinícola relativamente recente e com características climáticas particulares, como: altitude em torno de $1136 \mathrm{~m}$, proximidade do Oceano Atlântico (cerca de $150 \mathrm{~km}$ ) e latitude de 28 으. Essa região concentra $50,6 \%$ dos vinhedos de altitude do Estado, englobando o maior número de propriedades vitivinícolas (BORGHEZAN et al., 2011; VIANNA et al., 2016). Entretanto, é importante referir que a diversidade climática entre as regiões vitivinícolas muitas vezes expõe as plantas a condições ambientais bastante distintas com reflexos diretos sobre a produtividade e a qualidade dos frutos (NEIS et al., 2010; RADÜNZ et al., 2015). Portanto, o conhecimento dos estádios fenológicos ao longo de uma série histórica de ciclos é fundamental para caracterizar o comportamento das videiras em determinada região, pois permite modificar o manejo das plantas e programá-lo como forma de melhorar a produção (MANDELLI et al., 2003; OLIVEIRA et al., 2013). A caracterização das exigências térmicas das videiras por meio de um modelo de graus-dia (GD) é um método utilizado por muitos autores por ser confiável e eficiente (RADÜNZ et al., 2015; POLA et al., 2017; BRIXNER et al., 2010; ANZANELLO \& CHRISTO, 2019). Algumas pesquisas abordaram a fenologia da videira nas condições das regiões de altitudes de Santa Catarina, dentre elas as de BORGHEZAN et al. (2011), MALINOVSKI et al. (2012) e BRIGHENTI et al. (2013) e, entretanto, utilizaram poucos anos para a caracteri- zação fenológica dos cultivares.

Nos últimos anos foram introduzidas, comercialmente, na região de São Joaquim, diversas variedades viníferas com elevado potencial para a produção de vinhos de alta qualidade, como, por exemplo, 'Sauvignon Blanc', 'Montepulciano', 'Rebo', 'Sangiovese' entre outras. Considerando a importância econômica dessas variedades, é fundamental a compreensão dos seus comportamentos frente às condições térmicas disponíveis na região.

O objetivo deste trabalho foi caracterizar a fenologia, as necessidades térmicas e os indicadores de maturação das bagas de oito variedades viníferas durante uma série histórica entre 2013/14 a 2019/20 nas condições de São Joaquim, SC.

\section{Material e métodos}

A pesquisa foi realizada no município de São Joaquim, SC $\left(28^{\circ} 16^{\prime} 30^{\prime \prime}\right.$, ,

Recebido em 6/10/2020. Aceito para publicação em 16/2/2021.

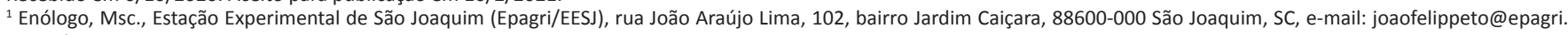
sc.gov.br.

${ }^{2}$ Engenheiro-agrônomo, Dr., Epagri/EEV, email: caliari@epagri.sc.gov.br

${ }^{3}$ Engenheiro-agrônomo, Msc., Epagri/EESJ, email: brighent@epagri.sc.gov.br

${ }^{4}$ Engenheiro-agrônomo, Dr., Epagri/Cepaf, email: cristiano@epagri.sc.gov.br 
$49^{\circ} 56^{\prime} 09^{\prime \prime}$ W, a $1.403 \mathrm{~m}$ de altitude). O vinhedo (Vitis vinifera L.) foi implantado em 2006, com espaçamento de 3,00m entre linhas e 1,50m entre plantas, e sistema de condução tipo espaldeira. As variedades avaliadas foram: 'Verdichio', 'Sauvignon Blanc', 'Garganega' e 'Manzoni Bianco' (brancas); 'Sanngiovese', 'Sagrantino', 'Montepulciano' e 'Rebo' (tintas). As datas de ocorrência dos principais estádios fenológicos: início da brotação (IB) plena floração (PF) e mudança de cor (MC). A análise das variedades foi baseada na escala proposta por Baillod \& Baggiolini (1993).

Os dados meteorológicos foram obtidos através da Estação Meteorológica Automática localizada junto ao vinhedo. As variáveis meteorológicas utilizadas foram: temperaturas do ar média, máxima e mínima $\left({ }^{\circ} \mathrm{C}\right)$, as quais foram utilizadas nos modelos de soma térmica expressos em graus-dia (GD) conforme WINKLER (1980). Estes GD foram calculados a partir dos valores obtidos pelas equações: $\mathrm{GD}=\Sigma(\mathrm{Tm}-\mathrm{Tb})+[(\mathrm{TM}-\mathrm{Tm}) / 2]$, quando $\mathrm{Tm}>\mathrm{Tb} ; \mathrm{GD}=\Sigma[(\mathrm{TM}-\mathrm{Tb}) 2 / 2 *(\mathrm{TM}-$ $\mathrm{Tm})$ ], quando $\mathrm{Tm} \leq \mathrm{Tb}$ e; $\mathrm{GD}=0$, quando $T b \geq T M$; em que $G D$ são os graus-dia; TM a temperatura máxima, em ${ }^{\circ} \mathrm{C}$; $\mathrm{Tm}$ a temperatura mínima, em ${ }^{\circ} \mathrm{C}$ e $\mathrm{Tb}$ a temperatura-base de $10^{\circ} \mathrm{C}$.

O delineamento experimental foi inteiramente casualizado, com cinco repetições de oito plantas de cada variedade, totalizando 320 plantas. Foram avaliados os ciclos entre 2013/14 e 2019/20. Por ocasião das colheitas, os mostos foram analisados para a determinação da acidez total titulável (ATT), sólidos solúveis totais (SST) e $\mathrm{pH}$, de acordo com a metodologia descrita por Rizzon (2010). A ordenação dos dados obtidos foi realizada com o auxílio de planilhas Excel, sendo calculadas, para cada variável, as médias e os desviospadrão amostrais de todos os ciclos e variedades estudadas. Os dados foram submetidos à análise de variância após verificadas e atendidas as pressuposições de homogeneidade de variâncias e a distribuição normal dos resíduos. $\mathrm{Na}$ análise, os valores das variáveis são as médias das repetições dentro de cada ano e os anos foram considerados as repetições para cada variedade. Em caso de efeito significativo, as médias foram comparadas pelo teste de Scott-Knott.
Todas as análises foram realizadas com o ambiente R (R CORE TEAM, 2020), com nível de significância de $5 \%$.

\section{Resultados e discussão}

A Tabela 1 apresenta as datas de ocorrência dos principais estádios fenológicos das variedades estudadas. No que se refere ao IB, as mais precoces foram 'Rebo' (29/08) e 'Sangiovese' (09/09), seguidas pelas variedades 'Verdichio' (11/09), 'Manzoni Bianco' (13/09), 'Sagrantino' (16/09), 'Sauvignon Blanc' (17/09), 'Montepulciano' (21/09) e, finalmente, 'Garganega' (24/09) (Tabela 1). Resultados similares foram obtidos por Brighenti (2014) estudando o comportamento fenológico das variedades 'Verdichio', 'Montepulciano', 'Sagrantino' e 'Sangiovese' em São Joaquim, SC. As maiores variabilidades do IB foram observadas nas variedades 'Rebo', Manzoni Bianco e Garganega. Na 'Rebo', por exemplo, houve uma diferença de 22 dias entre os anos de 2015/16 em relação a 2018/19 (Tabela 1). Essa diferença pode ser explicada pela influência das condições meteorológicas específicas de cada ano. Paradoxalmente, essas mesmas condições parecem não afetar de maneira uniforme todas as variedades, uma vez que a IB da 'Montepulciano', por exemplo, teve uma variabilidade de apenas 3 dias nos mesmos ciclos. Considerando que as características fenotípicas de cada variedade são distintamente afetadas, apesar de estarem sujeitas às mesmas condições climáticas, Borghezan et al. (2011), sustentam que outros fatores devem ser considerados, como a data da poda e as condições dos invernos.

A diferença entre o IB da variedade mais precoce, ('Rebo', 29/08) em relação à mais tardia ('Garganega', 24/09), foi de 26 dias. Com base nessa diferença, as variedades podem ser classificadas em a) precoces: as que iniciam a brotação até o dia 9 de setembro; b) intermediárias: quando iniciam a brotação entre 10 e 17 de setembro e c) tardias: quando iniciam a brotação após o dia 18 de setembro. Portanto, são variedades de brotação precoce a 'Rebo' e a 'Sangiovese'; de brotação intermediária a 'Verdichio', 'Manzoni Bianco',
'Sagrantio' e 'Sauvignon Blanc' e tardias a 'Montepulciano' e a 'Garganega'.

A classificação da precocidade com base na época do IB é importante para os produtores, pois ela fornece subsídios para a escolha de variedades levando em conta a disponibilidade térmica do local do cultivo. Desta forma, variedades mais precoces podem ser cultivadas em locais com baixo risco de geadas primaveris, enquanto aquelas com brotação mais tardia podem ser a melhor escolha para locais com menor risco. É importante referir que na região de São Joaquim ocorrem, com frequência, geadas capazes de causar fortes declínios quantitativos nas produções. Por esta razão, o atraso no IB pode significar uma vantagem adaptativa importante em termos de estabilidade produtiva (MANDELLI et al., 2003). A PF foi observada no mês de novembro para a maioria das variedades, exceto a 'Montepulciano' e a 'Garganega', que floresceram plenamente no início de dezembro. Constataram-se 19 dias de diferença entre a variedade com floração mais precoce ('Rebo', 13/11) em relação à mais tardia ('Montepulciano', 02/12) (Tabela 1). Entretanto, no que se refere à $M C$, foi verificada, em média, uma diferença de 35 dias entre a variedade com maturação mais precoce ('Manzoni Bianco', 20/01) e a mais tardia ('Garganega', 24/02) (Tabela 1). Em geral, as variedades mais precoces em sua brotação tendem a ser as mais precoces na floração. Por outro lado, quanto à maturação das bagas, nem todas as variedades demostraram essa tendência. Por exemplo, a 'Manzoni Bianco' é classificada como intermediária em termos de IB (13/09), porém, em média, foi a primeira a ser colhida dentre as variedades estudadas (15/03). Esta mesma observação também pode ser feita para a 'Sauvignon Blanc' que, embora seja intermediária em termos de IB (17/09), teve sua coIheita no dia (20/03), sendo a segunda a ser colhida dentre as oito variedades. No que se refere à maturação das uvas, Rosier (2003) sustenta que as condições térmicas da região de São Joaquim, SC, proporcionadas pelas temperaturas noturnas amenas, induzem à redução do metabolismo das plantas, retardando o amadurecimento dos frutos quando comparadas a outras regiões produto- 
Tabela 1. Datas de ocorrência das diferentes fases fenológicas de diferentes variedades de videira nos ciclos entre 2013/14 e 2019/20. São Joaquim, SC

Table 1. Dates of occurrence of the different phenological phases of different grape varieties in the cycles between 2013/14 and $2019 / 20$. São Joaquim, SC

Estádios

Médias das datas de ocorrência dos estádios fenológicos

\begin{tabular}{|c|c|c|c|c|c|c|c|c|c|}
\hline \multicolumn{10}{|c|}{ Fenológicos } \\
\hline & Safra & VD* & SB* & GG* & MB* & SG* & SN* & MP* & RB* \\
\hline \multirow{7}{*}{ IB } & $2013 / 14$ & 09/set & $16 /$ set & 05/out & $13 /$ set & $20 /$ set & $23 /$ set & $18 /$ set & 26/ago \\
\hline & $2014 / 15$ & $10 /$ set & $16 /$ set & $30 /$ set & $11 /$ set & 09/set & $20 /$ set & $30 /$ set & $01 /$ set \\
\hline & $2015 / 16$ & 05/set & $18 /$ set & $22 /$ set & $13 /$ set & $06 /$ set & $15 /$ set & $22 /$ set & 17/ago \\
\hline & $2016 / 17$ & $17 /$ set & $17 /$ set & $23 /$ set & $21 /$ set & 14 /set & 14 /set & $22 /$ set & $03 /$ set \\
\hline & $2017 / 18$ & $06 /$ set & 14 /set & $16 /$ set & $02 /$ set & $02 /$ set & 06/set & $12 /$ set & 28/ago \\
\hline & 2018/19 & $20 /$ set & $27 /$ set & $30 /$ set & $24 /$ set & $10 /$ set & $20 /$ set & $25 /$ set & $08 /$ set \\
\hline & $2019 / 20$ & $11 /$ set & $13 /$ set & $18 /$ set & $13 /$ set & $06 /$ set & $15 /$ set & $21 /$ set & $02 /$ set \\
\hline Médias & & 11-set & 17-set & 24-set & 13-set & 9-set & 16 -set & 21-set & 29-ago \\
\hline DP* & & 6 & 5 & 7 & 7 & 6 & 6 & 6 & 7 \\
\hline \multirow{7}{*}{ PF } & $2013 / 14$ & $27 /$ nov & 01/dez & 09/dez & $22 /$ nov & $25 /$ nov & 03/dez & 05/dez & $24 /$ nov \\
\hline & $2014 / 15$ & $13 /$ nov & $20 /$ nov & $28 /$ nov & $10 /$ nov & $12 /$ nov & $24 /$ nov & $27 /$ nov & $10 /$ nov \\
\hline & $2015 / 16$ & $20 /$ nov & $20 /$ nov & $27 /$ nov & $10 /$ nov & $14 /$ nov & $23 /$ nov & $28 /$ nov & $12 /$ nov \\
\hline & $2016 / 17$ & 01/dez & 01/dez & 05/dez & $28 /$ nov & $24 /$ nov & $05 / \mathrm{dez}$ & $12 / \mathrm{dez}$ & $28 /$ nov \\
\hline & $2017 / 18$ & $07 /$ nov & $08 /$ nov & $25 /$ nov & $28 /$ nov & $04 /$ nov & $08 /$ nov & $25 /$ nov & 22/out \\
\hline & $2018 / 19$ & $26 /$ nov & $28 /$ nov & $12 / \mathrm{dez}$ & $25 /$ nov & $23 /$ nov & $23 /$ nov & 08/dez & $20 /$ nov \\
\hline & $2019 / 20$ & $13 /$ nov & $16 /$ nov & $27 /$ nov & $06 /$ nov & $06 /$ nov & $23 /$ nov & 01/dez & $06 /$ nov \\
\hline Médias & & 19-nov & 22-nov & 1-dez & 18-nov & 15-nov & 24-nov & 2-dez & 13-nov \\
\hline DP* & & 9 & 9 & 7 & 9 & 9 & 9 & 6 & 13 \\
\hline \multirow{7}{*}{ MC } & $2013 / 14$ & $09 / \mathrm{fev}$ & $07 / \mathrm{fev}$ & $20 / \mathrm{fev}$ & 29/jan & $12 / \mathrm{fev}$ & $12 / \mathrm{fev}$ & $24 / \mathrm{fev}$ & $13 / \mathrm{fev}$ \\
\hline & $2014 / 15$ & $02 / \mathrm{fev}$ & 25/jan & $15 / \mathrm{fev}$ & 15/jan & 30/jan & 31/jan & $15 / \mathrm{fev}$ & $21 /$ jan \\
\hline & $2015 / 16$ & $11 /$ fev & 29/jan & $22 / \mathrm{fev}$ & $22 / j a n$ & $05 / \mathrm{fev}$ & $03 / \mathrm{fev}$ & $11 /$ fev & $27 /$ jan \\
\hline & $2016 / 17$ & $17 / \mathrm{fev}$ & $11 / \mathrm{fev}$ & $04 / \mathrm{mar}$ & $10 /$ fev & $15 / \mathrm{fev}$ & $14 / \mathrm{fev}$ & $03 / \mathrm{mar}$ & $09 / \mathrm{fev}$ \\
\hline & $2017 / 18$ & $10 /$ fev & $11 / \mathrm{fev}$ & $02 / \mathrm{mar}$ & 17/jan & $01 / \mathrm{fev}$ & 22/jan & $27 / \mathrm{fev}$ & 20/jan \\
\hline & $2018 / 19$ & $06 / \mathrm{fev}$ & $05 / \mathrm{fev}$ & $26 / \mathrm{fev}$ & $26 / \mathrm{dez}$ & $12 / \mathrm{fev}$ & $10 /$ fev & $28 / \mathrm{fev}$ & $25 /$ jan \\
\hline & $2019 / 20$ & $11 /$ fev & 23/jan & $25 / \mathrm{fev}$ & 22/jan & 20/jan & 24/jan & $24 / \mathrm{fev}$ & $14 /$ jan \\
\hline Médias & & 9-fev & 2-fev & 24-fev & 20-jan & 4-fev & 3-fev & 22-fev & 27-jan \\
\hline DP* & & 5 & 8 & 6 & 14 & 9 & 9 & 7 & 11 \\
\hline \multirow{7}{*}{ COL } & $2013 / 14$ & $12 /$ mar & $12 /$ mar & $22 / a b r$ & $12 /$ mar & 07/abr & 08/abr & $22 / a b r$ & $08 / a b r$ \\
\hline & $2014 / 15$ & $23 / \mathrm{mar}$ & $23 / \mathrm{mar}$ & $22 / a b r$ & $16 /$ mar & $22 / a b r$ & $22 / a b r$ & $30 / a b r$ & $15 / a b r$ \\
\hline & $2015 / 16$ & $28 /$ mar & $16 /$ mar & $05 / a b r$ & 09/mar & $28 / \mathrm{mar}$ & $28 /$ mar & $05 / a b r$ & $22 / \mathrm{mar}$ \\
\hline & $2016 / 17$ & $10 / a b r$ & $29 /$ mar & $02 / a b r$ & 29/mar & $19 / a b r$ & $25 / a b r$ & $19 / a b r$ & $19 / a b r$ \\
\hline & $2017 / 18$ & $21 /$ mar & 03/abr & $23 / a b r$ & $21 /$ mar & $21 / \mathrm{mar}$ & $10 / a b r$ & $23 / a b r$ & - \\
\hline & $2018 / 19$ & $08 / a b r$ & $20 /$ mar & $24 / a b r$ & $20 /$ mar & $27 / \mathrm{mar}$ & $04 / a b r$ & $24 / a b r$ & - \\
\hline & $2019 / 20$ & $13 / a b r$ & 09/mar & 14/abr & 03/mar & $23 / \mathrm{mar}$ & $24 / \mathrm{mar}$ & $30 /$ mar & $23 / \mathrm{mar}$ \\
\hline Médias & & 29-mar & 20-mar & 16-abr & 15-mar & 3-abr & 8-abr & $17-a b r$ & $5-a b r$ \\
\hline DP* & & 12 & 9 & 9 & 9 & 13 & 12 & 11 & 13 \\
\hline
\end{tabular}

*VD = Verdicchio; SB = Sauvignon Blanc; GG = Garganega; MB = Manzoni Bianco; SG = Sangiovese; SN = Sagrantino; MP = Montepulciano; $\mathrm{RB}=$ Rebo

$(* *)$ DP $=$ Desvios Padrão $(* *) D P=$ Standard Deviation 
ras do país. Sendo assim, o deslocamento do ciclo vegetativo é o responsável pelas particularidades dos vinhos desta região, como a manutenção da acidez, mesmo nas bagas maduras. Estas observações são corroboradas pelos estudos realizados por Silva et al. (2009) e Borghezan et al. (2011). Quanto ao número de dias para completar os subperíodos fenológicos, as variedades apresentaram resultados bastante diversos (Figura 1). Foram necessários, em média, 183 e 184 dias, respectivamente, para as mais precoces 'Manzoni Bianco' e 'Sauvignon Blanc', enquanto para as mais tardias, como a 'Sagrantino', 'Montepulciano' e 'Rebo', foram necessários 206, 208 e 214 dias, respectivamente. Resultados semelhantes foram obtidos por Malinovski et al. (2016) ao estudarem a fenologia de variedades autóctones italianas na região de Água Doce, SC. A análise cronológica de cada subperíodo não evidenciou diferenças significativas no número de dias contabilizados entre o IB e a PF em nenhuma variedade (Figura 1). Entretanto, essa mesma análise mostra diferenças significativas nos subperíodos compreendidos entre a
PF e a MC. Essas diferenças podem ser explicadas pela variabilidade genética e, consequentemente, pelas diferentes respostas das plantas às condições impostas pelo ambiente.

Relativamente aos requerimentos térmicos necessários para completar os subperíodos entre as brotações e as colheitas, os maiores valores foram observados nas variedades 'Sagrantino', (1399GD), 'Montepulciano', (1.424GD) e 'Rebo', (1.438GD). Os menores requerimentos foram 'Manzoni Bianco', (1268GD); 'Sauvignon Blanc', (1.270GD) 'Verdicchio', (1.362GD). Radünz et al. (2015), estudando os requerimentos térmicos de diversas variedades nas condições da Campanha gaúcha, obtiveram 1.759GD para 'Sauvignon Blanc', valor este $39 \%$ superior ao observado para a mesma variedade em São Joaquim (Figura 2). Essa diferença encontra explicação nas temperaturas mais altas registradas na Campanha Gaúcha em relação a São Joaquim. As análises dos requerimentos térmicos necessários para completar os subperíodos entre o início da brotação até a plena floração não evidenciaram diferenças significati- vas entre as variedades, exceto para a 'Manzoni Bianco', cujos requerimentos térmicos se mostraram significativamente menores em relação às demais $(463,48 \mathrm{GD})$. Entretanto, no subperíodo entre a MC e a colheita, foram constatadas diferenças significativas nos requerimentos térmicos entre as variedades 'Rebo' (474,10GD), 'Sagrantino' (451,32GD), 'Sangiovese' (406,96GD) e 'Manzoni Bianco' (439,00GD), sendo consideradas, neste estudo, como as mais exigentes termicamente (Figura 2). Considerando as condições climáticas de São Joaquim, onde os verões são amenos, é relevante notar que as variedades termicamente mais exigentes podem representar desafios fitotécnicos importantes para a maturação. Sendo assim, devem ser priorizadas ações de manejo do dossel, como a desfolha precoce, o desponte e a adequada exposição solar dos cachos.

Na Tabela 2 estão apresentados os indicadores da maturação. Os valores dos SST foram maiores nas variedades 'Rebo' (22,43 Brix), 'Sagran-

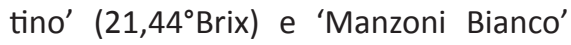
$\left(21,34^{\circ} \mathrm{Brix}\right)$. Esses valores são bons in-

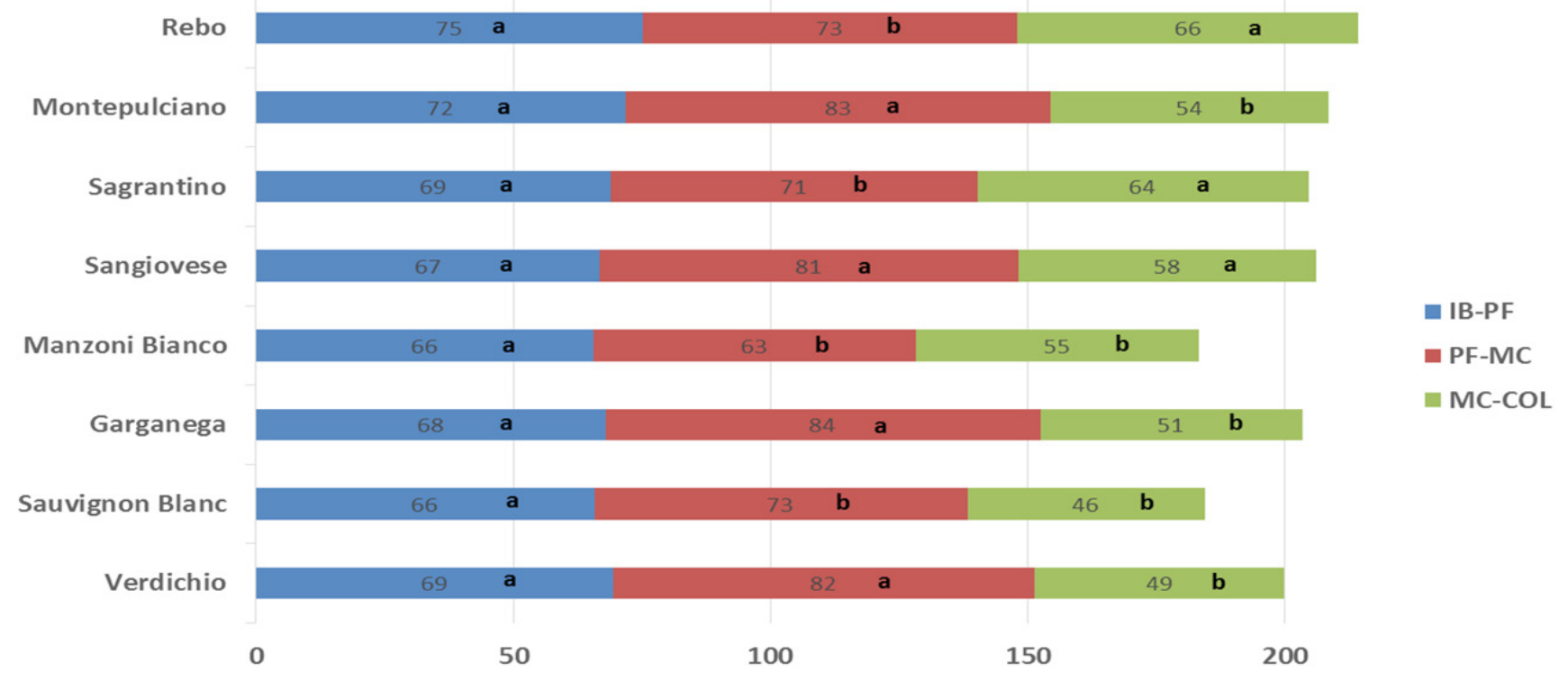

Dias para completar os subperíodos

Figura 1. Médias dos números de dias necessários para completar os subperíodos entre o início da brotação (IB), pleno florescimento (PF), mudança de cor (MC) e colheita (COL) de oito variedades de videira cultivadas em São Joaquim, SC entre 2013/14 e 2019/20. * Médias seguidas de mesma letra nas colunas não diferem pelo teste de Scott-Knott a $5 \%$ de probabilidade de erro

Figure 1. Averages of the number of days required to complete the subperiods between the beginning of sprouting (IB), full flowering (PF), color change (MC) and harvest (COL) of eight grape varieties grown in São Joaquim, SC between 2013/14 and 2019/20. * Averages followed by the same letter in the columns do not differ by the Scott-Knott test at 5\% probability of error 
Montepulciano
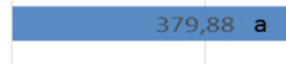
$348,70 \quad b$

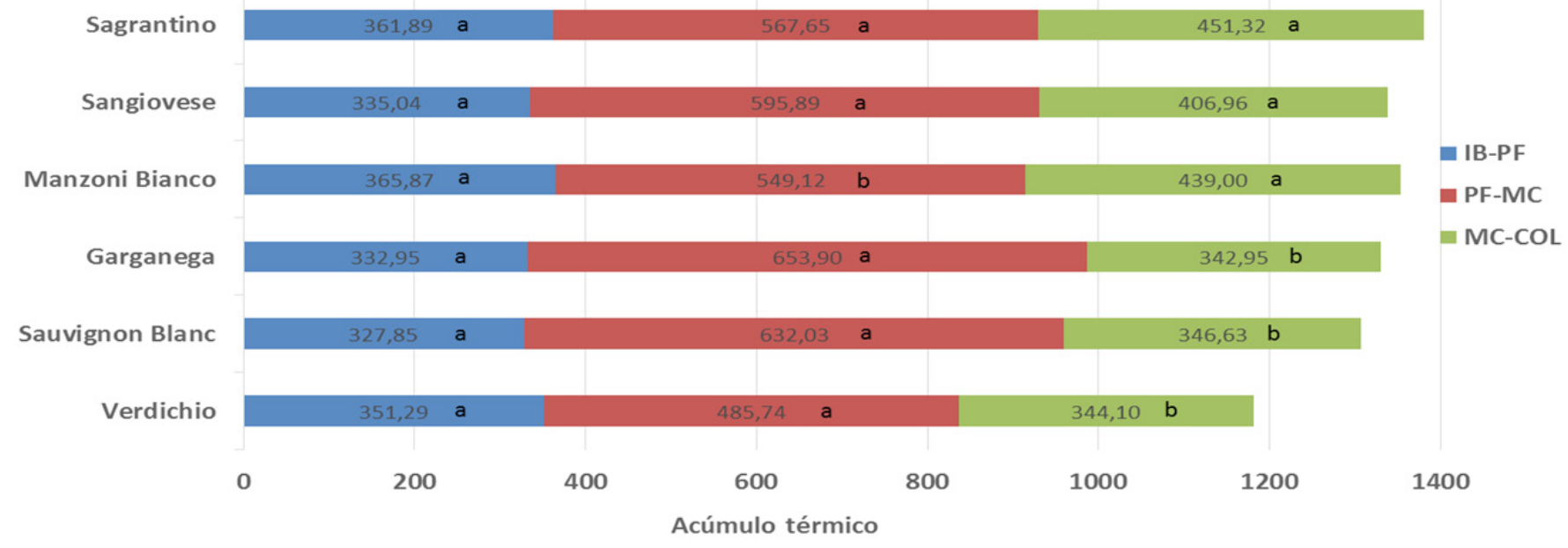

Figura 2. Médias dos acúmulos térmicos (graus-dia) necessários para completar os subperíodos entre o início da brotação (IB), pleno florescimento (PF), mudança de cor (MC) e colheita (COL) de oito variedades de videira cultivadas em São Joaquim, SC entre 2013/14 e 2019/20.

* Médias seguidas de mesma letra nas colunas não diferem pelo teste de Scott-Knott a 5\% de probabilidade de erro

Figure 2. Averages of thermal accumulations (degree-days) necessary to complete the subperiods between the beginning of sprouting (IB), full flowering (PF), color change (MC) and harvest (COL) of eight grape varieties grown in São Joaquim-SC between 2013/14 and 2019/20. * Averages followed by the same letter in the columns do not differ by the Scott-Knott test at 5\% probability of error

dicadores do desempenho qualitativo das variedades nas condições de São Joaquim, SC. Entretanto, todas as amostras apresentaram SST adequados para a elaboração de vinhos finos de qualidade (19,0 a 22,0 $0^{\circ}$ Brix).

Quanto à ATT, as médias obtidas não mostraram diferenças significativas entre si (Tabela 2). A ATT corresponde à soma dos ácidos presentes na matriz ácida das bagas de uva, e os seus teores podem ser influenciados pelas características inerentes a cada variedade, mas principalmente pelo clima. Müller (2016), estudando o potencial enológico de 12 variedades viníferas durante a safra de 2015 na região da Campanha Gaúcha, onde a altitude varia entre $100 \mathrm{~m}$ e $300 \mathrm{~m}$, observou que a ATT da 'Montepulciano' chegou a 62,22 $\mathrm{meq} \mathrm{L}^{-1}$, enquanto que a 'Rebo' foi $60,56 \mathrm{meq} L^{1}$. Os teores de ATT das uvas avaliadas estão de acordo com os limites estabelecidos pela legislação setorial (40meq $\mathrm{L}^{-1} \mathrm{a}$ 130meq L $\mathrm{L}^{-1}$ ) (BRASIL, 2018), exceto para a variedade 'Garganega' que atingiu, em média, 155meq $\mathrm{L}^{1}$. Para essa variedade, recomenda-se a adoção de manejos fitotécnicos capazes de reduzir a acidez, como a adequada exposição solar dos cachos, além de evitar locais com in-
Tabela 2. Características físico-químicas das uvas oriundas de diferentes variedades de videira nos ciclos entre 2013/14 e 2019/2020. São Joaquim, SC

Table 2. Physico-chemical characteristics of grapes in the harvests of different grape varieties in the cycles between 2013/14 to 2019/20. São Joaquim, SC

\begin{tabular}{|c|c|c|c|c|c|c|}
\hline \multirow[b]{2}{*}{ Variedades } & \multicolumn{2}{|c|}{$\mathrm{SST}^{*}\left({ }^{\circ}\right.$ Brix $)$} & \multicolumn{2}{|l|}{$\mathrm{pH}$} & \multicolumn{2}{|c|}{ ATT* $^{*}\left(\mathrm{meq}^{-1}\right)$} \\
\hline & Médias (1) & DP* & Médias ${ }^{(1)}$ & DP* & Médias ${ }^{(1)}$ & DP* \\
\hline Verdicchio & $19,53 \mathrm{~b}$ & 1,82 & 2,92 a & 0,11 & $132,08 \mathrm{a}$ & 25,95 \\
\hline Sauvignon Blanc & $20,17 b$ & 1,32 & 2,99 a & 0,10 & 112,85 a & 10,07 \\
\hline Garganega & $19,90 \mathrm{~b}$ & 0,81 & 2,90 a & 0,10 & 155,48 a & 54,17 \\
\hline Manzoni Bianco & 21,34 a & 1,27 & $2,97 \mathrm{a}$ & 0,11 & 106,23 a & 18,53 \\
\hline Sangiovese & $19,02 \mathrm{~b}$ & 1,24 & 3,03 a & 0,10 & 119,72 a & 23,04 \\
\hline Sagrantino & 21,44 a & 1,03 & $2,97 \mathrm{a}$ & 0,09 & 131,81 a & 25,91 \\
\hline Montepulciano & $20,38 \mathrm{~b}$ & 1,78 & 2,98 a & 0,08 & 127,27 a & 23,48 \\
\hline Rebo & 22,43 a & 2,03 & 3,09 a & 0,13 & 111,44 a & 31,62 \\
\hline
\end{tabular}

(*) SST - Sólidos Solúveis Totais; ATT - Acidez Total Titulável; DP - Desvio Padrão (1) Médias seguidas de mesma letra nas colunas não diferem pelo teste de Scott-Knott a 5\% de probabilidade de erro

(*) SST - Total Soluble Solids; ATT - Total Titratable Acidity; SD - Standard Deviation (1) Averages followed by the same letter in the columns do not differ by the Scott-Knott test at 5\% probability of error

cidência de ventos frios, otimizando, assim, o acúmulo de graus-dia no nível dos cachos.

$\mathrm{O} \mathrm{pH}$ corresponde à concentração de hidrogênio iônico proveniente dos ácidos orgânicos, somada à concentração de potássio, representando a aci- dez real do vinho (GABAS et al., 1994; RIZZON \& MIELE, 2004). Da mesma forma que para a ATT, o pH sofre influência das condições climáticas, de forma que as uvas cultivadas em regiões de clima ameno ou frio, têm um pH mais baixo. De acordo com Müller (2016) o pH das \& 
variedades 'Montepulciano e 'Rebo' atingiu 4,02 e 4,07, respectivamente, durante a safra de 2015 na região da Campanha Gaúcha, enquanto nas condições de São Joaquim essas mesmas variedades chegam, em média, a 2,98 e 3,09, respectivamente. É importante salientar que as características da matriz ácida contribuem fortemente para os atributos de tipicidade gustativa denotada pela vivacidade e frescor dos vinhos, especialmente daqueles elaborados com variedades brancas.

\section{Conclusões}

- As variedades de videira avaliadas quanto à brotação podem ser classificadas em precoces ('Rebo' e 'Sangiovese'); intermediárias ('Verdichio', 'Manzoni Bianco', 'Sagrantino' e 'Sauvignon Blanc') e tardias ('Montepulciano' e 'Garganega').

- O ciclo vegetativo médio das variedades foi de 200 dias, sendo a 'Rebo' a variedade com ciclo mais longo (214 dias e 1.493GD) e a 'Manzoni Bianco' a com ciclo mais curto (184 dias e 1.353GD).

- As videiras estudadas produziram frutos com acidez total titulável, sólidos solúveis totais e $\mathrm{pH}$ adequados para a elaboração de vinhos finos, com destaque qualitativo para a 'Rebo' dentre as tintas e a 'Manzoni Bianco' dentre as brancas.

\section{Referências}

ANZANELLO, R.; CHRISTO, M. C. Temperatura base inferior, soma térmica e fenologia de cultivares de videira e quivizeiro. Revista de Ciências Agroveterinárias, Lages, v. 18 n. 3, p. 313-322, 2019.

BAILLOD, M.; BAGGIOLLINI, M. Les stades repères de la vigne. Revue Suisse de Viticulture, Arboriculture. Horticulture. v. 25, n. 1, p. 7-9, 1993.

BORGHEZAN, M., GAVIOLI, O, PIT, F. A.; DA SILVA, A. L. Comportamento vegetativo e produtivo da videira e composição da uva em São Joaquim, Santa Catarina. Pesq. agropec. bras., Brasília, v.46, n.4, p.398-405, 2011.

BRASIL. Ministério da Saúde. Secretaria de
Vigilância Sanitária. (2018, março 9). Complementação dos padrões de identidade e qualidade do vinho e dos derivados da uva e do vinho (Instrução Normativa no 14, de 08 de fevereiro de 2018). Diário Oficial [da] República Federativa do Brasil, Brasília, Seção 1, p. 4-6.

BRIGHENTI, A. F., BRIGHENTII, E., BONIN, V.; RUFATO, L. Caracterização fenológica e exigência térmica de diferentes variedades de uvas viníferas em São Joaquim, Santa Catarina - Brasil. Ciência Rural, Santa Maria, v.43, n.7, p.1162-1167, 2013.

BRIGHENTI, A. F., DA SILVA, A. L., BRIGHENTI, E., PORRO, D., STEFANINI, M. Desempenho vitícola de variedades autóctones italianas em condição de elevada altitude no Sul do Brasil. Pesquisa agropecuária brasileira, Brasília, v.49, n.6, p.465-474, 2014.

BRIXNER, G. F., MARTINS, C. R., AMARAL, U.; KÖPP, L. M., OLIVEIRA, D. B. Caracterização fenológica e exigência térmica de videiras $V i$ tis vinifera, cultivadas no município de Uruguaiana, na região da fronteira oeste - RS. Revista FZVA, Uruguaiana, v.17, n.2, p.221233. 2010.

GABAS, N., RATSIMBA, B., GERBAUD, V. Les sels tartriques dans les vins: solubilité et sursaturation. In: La microbiologie des vins mousseux: la stabilisation des vins - mécanismes et évaluation. Toulouse : Lallemand, p. 95-98, 1994.

MALINOVSKI, L. I., VIEIRA, H. J., CAMARGO, C. G., STEFANINI, M., DA SILVA, A. L. Climate and Phenology: Behavior of Autochthonous Italian Grapevine Varieties in the Uplands of Southern Brazil. Journal of Agricultural Science, v.8, n.5; p. 26-33, 2016.

MALINOVSKI, L. I., WELTER, L. J.; BRIGHENTI, A. F., VIEIRA, H. J., GUERRA, M. P., DA SILVA, A. L. Highlands of Santa Catarina/Brazil: a Region with High Potential for Wine Production. Acta Horticulturae 931:433-440. 2012. Disponível em: www.researchgate.net/publication/275274891. Acesso em: 20 ago. 2020.

MANDELLI, F., BERLATO, M. A., TONIETTO, J.; BERGAMASCHI, H., Fenologia da videira na serra gaúcha. Pesquisa Agropecuária Gaúcha, v. 9, n. 1-2, p. 129-144, 2003.

MÜLLER, R.E., Potencial enológico de novas variedades cultivadas na campanha gaúcha. 2016. 73f. Mestrado (Mestrado em Ciência e Tecnologia de Alimentos) Programa de Pós-Graduação em Tecnologia de Alimentos da Universidade Federal do Rio Grande do Sul, Porto Alegre, RS, 2016.

NEIS, S. et al. Caracterização fenológica e requerimento térmico para a videira Niágara Rosada em diferentes épocas de poda no sudoeste goiano. Revista Brasileira de Fruticultura, v.32, p.931-937, 2010.

OLIVEIRA, I. V. de M., LOPES, P. R. C., DA SILVA-MATOS, R. R. S. Caracterização fenológica e frutificação efetiva de macieiras 'Daiane' sob condições semiáridas no nordeste do Brasil. Revista de Ciências Agroambientais, Alta Floresta, v.11, n.2, p.153-158, 2013.

POLA, A. C., BRUNA, E. D., BACK, A. J., MORETO, A. L. Estimativa das datas de brotação, plena floração e colheita da videira variedade Goethe em Urussanga-SC, Brasil. Revista de Ciências Agrárias, v.40, n.1, p. 236-245, 2017.

R Core Team (2020). R: A language and environment for statistical computing. R Foundation for Statistical Computing, Vienna, Austria. https://www.R-project.org/

RADÜNZ, A. L., SCHÖFFEL; E.R., BORGES, C.T., MALGARIM, M.B., PÖTTER, G.H. Necessidades térmicas de videiras na região da Campanha do Rio Grande do Sul - Brasil. Ciência Rural, Santa Maria, v.45, n.4, p.626632, 2015.

RIZZON, L. A.; MIELE, A. Avaliação da cv. Tannat para elaboração de vinho tinto. Ciência e Tecnologia de Alimentos., Campinas, v.24 n.2 p.223-229, 2004. 116p.

RIZZON, L. A. Metodologia para análise de vinho. Brasília, DF, Embrapa Informação Tecnológica; Bento Gonçalves: Embrapa Uva e Vinho, 2010.

ROSIER, J.P. Novas regiões: vinhos de altitude no sul do Brasil. In: CONGRASSO BRASILEIRO DE VITICULTURA E ENOLOGIA, 10.,2003, Bento Gonçalves, 2003. p.137.

SILVA, L.C. da, RUFATO, L., KRETZSCHMAR, A.A., MARCON FILHO, J.L. Raleio de cachos em vinhedos de altitude e qualidade do vinho da cultivar Syrah. Pesquisa Agropecuária Brasileira, v.44, p.148-154, 2009.

VIANNA, L.F., MASSIGNAN, A.M., PANDOLFO, C., DORTZBACH, D., VIEIRA, V.F. Caracterização agronômica e edafoclimática dos vinhedos de elevada altitude. Revista de Ciências Agroveterinárias, Lages, v.15, n.3, p.215-226, 2016.

WINKLER, A.J. Viticultura. 6.ed. México: Editorial Continental, 1980. 791p. 